\title{
Conflito Conjugal e Estratégias de Resolução: Uma Revisão Sistemática da Literatura
}

\author{
Crístofer Batista da Costa ${ }^{1}$ \\ Programa de Pós-Graduação em Psicologia da Universidade do Vale do Rio dos Sinos, \\ São Leopoldo, RS, Brasil \\ Cláudia Mara Boseto Cenci \\ Curso de Psicologia da Faculdade Meridional IMED, Passo Fundo, RS, Brasil \\ Clarisse Pereira Mosmann \\ Programa de Pós-Graduação em Psicologia da Universidade do Vale do Rio dos Sinos,
}

São Leopoldo, RS, Brasil

\begin{abstract}
Resumo
Os conflitos conjugais e as estratégias de resolução são pesquisados na literatura internacional há mais de duas décadas. Nesse sentido, analisar a literatura nacional e internacional acerca do conflito conjugal e das estratégias de resolução de conflito empregadas pelos cônjuges, foi o objetivo deste estudo. Tratase de uma revisão sistemática qualitativa entre o período de 2004/2013. O estudo foi realizado em dez bases de dados através dos descritores conflito conjugal $A N D$ casamento e resolução de problemas $A N D$ casamento e submetidos aos procedimentos de análise de conteúdo. Na primeira etapa foram selecionados 567 artigos e, destes, 30 compõem esta revisão por atenderem aos critérios de exclusão e inclusão definidos a priori. Os resultados dos estudos apontaram que as pesquisas sobre as estratégias de resolução se sobressaíram em detrimento dos motivos dos conflitos conjugais. Identificou-se que as variáveis sexo, características individuais, tempo de união e processos interacionais estão associadas à utilização dos tipos de estratégias, construtivas e destrutivas. Estudos sobre o poder de predição dessas variáveis sobre as estratégias de resolução precisam ser mais explorados, especialmente, no contexto brasileiro, em que há escassez de pesquisas sobre a temática.
\end{abstract}

Palavras-chave: Conflito conjugal, casamento, resolução de problemas.

\section{Marital Conflict and Resolution Strategies: A Systematic Literature Review}

\begin{abstract}
Marital conflicts and resolution strategies are studied in the literature for over two decades. Accordingly, the purpose of this article was to analyze the national and international literature on marital conflict and resolution strategies employed by the spouses. This is a qualitative systematic review of the period between 2004/2013. The study was conducted in nine databases through descriptors marital conflict AND marriage and problem resolution AND marriage subjected to content analysis procedures. In the first step, 567 articles were selected. 30 of these are part of this systematic review compose since they obey the operational exclusion and inclusion criteria defined a priori. The studies point out that research
\end{abstract}

Endereço para correspondência: Rua Coronel Corte Real, 913/204, Petrópolis, Porto Alegre, RS, Brasil 90630080. E-mail: cristoferbatistadacosta@gmail.com, claudia.cenci@imed.edu.bre clarissemosmann@gmail.com 
on resolution strategies stood out over the grounds of marital conflict. It was identified that the gender, individual characteristics, time of unity and interaction processes are associated with the use of the types of strategies, constructive and destructive. Studies on the predictive power of these variables on the resolution strategies need further exploration, especially in the Brazilian context, where there are few investigations on the subject.

Keywords: Marital conflict, marriage, problem solving.

\section{Conflicto Marital y Estrategias de Resolución: Una Revisión Sistemática de la Literatura}

\section{Resumen}

Conflictos maritales y sus estrategias de resolución son estudiados en la literatura por más de dos décadas. En este sentido, el objetivo de este artículo fue analizar la literatura nacional e internacional y las estrategias de resolución de conflictos maritales. Se trata de una revisión sistemática cualitativa entre el período de 2004/2013. El estudio se realizó en nueve bases de datos por medio de descriptores conflicto marital y matrimonio y resolución de problemas y matrimonio sometidos al análisis de contenido cualitativo. En el primer paso fueron seleccionados 567 artículos y, de éstos, 30 componen esta revisión sistemática de la literatura por cumplir con los criterios de exclusión y inclusión establecidos a priori. Los resultados de los estudios enseñan que las investigaciones acerca de las estrategias de resolución sobresalen las de razones de los conflictos maritales. Se identificó que las variables sexo, características individuales, tiempo de unión y procesos interacciónales están asociadas a utilización de los tipos de estrategias, constructivas e destructivas. Estudios acerca del poder predictivo de esas variables en las estrategias de resolución necesitan mayor explotación, en especial, en el contexto brasileño, en lo cual hay escasez de investigaciones sobre el tema.

Palabras clave: Conflicto marital, matrimonio, resolución de problemas.

Os conflitos conjugais vêm sendo estudados no contexto internacional há pelo menos duas décadas. Trata-se de um fenômeno multidimensional, envolvendo a frequência com que ocorre, a intensidade das discussões, o conteúdo que fez emergir a situação conflituosa, o reconhecimento dos cônjuges sobre a existência ou não de problemas, e as estratégias utilizadas para resolvê-los (Benetti, 2006; Cummings, 1998).

Situações de conflito na conjugalidade acontecem devido ao processo de adaptação, sincronia e amadurecimento da relação ao longo do tempo (Bertoni \& Bodenmann, 2010). No entanto, os conflitos poderão caracterizar-se, predominantemente, por interações de desacordo e invalidação ou por tentativas de reparar os problemas e validar os sentimentos e os pensamentos do cônjuge. Desse modo, o tipo de interação mais frequente entre os parceiros durante o conflito, indicará se ele será mais negativo e conflituoso ou positivo e facilitador à resolução dos problemas (Gottman, 1998).

Os desacordos conjugais ocorrem por diferentes motivos e podem estar relacionados às divergências sobre a educação dos filhos, o tempo que os parceiros ficam juntos, as finanças, a divisão das tarefas domésticas, as questões legais, entre outros motivos (Bolze, Schmidt, Crepaldi, \& Vieira, 2011). Os problemas podem envolver também a consistência do contrato conjugal (Tavora, 2009), a forma de se comunicar (Torossian, Heleno, \& Vizzotto, 2009), as características individuais como temperamento e autoestima, e os padrões de interação entre os cônjuges (Silva \& Vandenberghe, 2009).

Além disso, os contextos sociais e a cultura são fundamentais à compreensão dos conflitos no casamento (Garcia \& Tassara, 2001) visto que 
interferem na percepção e no papel de homens e mulheres na conjugalidade (Benetti, 2006; Giddens, 1993). Os reflexos dessas mudanças foram apontadas recentemente por Dush e Taylor (2012). De acordo com os pesquisadores, os casais que tomam as decisões em conjunto e compartilham as responsabilidades da vida conjugal e familiar apresentam menores níveis de conflito e maiores níveis de satisfação conjugal. Mostra-se importante analisar e discutir as mudanças socioculturais nos contextos em que os conflitos ocorrem, sob a premissa de que influenciam a forma de pensar das pessoas, as expectativas em torno da conjugalidade e a postura que os parceiros íntimos assumem em suas relações amorosas (Benetti, 2006; Dush \& Taylor, 2012; Garcia \& Tassara, 2001; Giddens, 1993).

Um estudo realizado em Santa Catarina, em 2013, objetivou caracterizar os relacionamentos conjugais e as estratégias de resolução de conflito de 200 casais de classe média. Os resultados indicaram altos níveis de satisfação conjugal e utilização de estratégias de negociação frente aos conflitos, mas também, emprego de estratégias de esquiva, agressão psicológica menor e contextos de reciprocidade negativa (Bolze, Schmidt, Crepaldi, \& Vieira, 2013). O estudo encontrou correlação entre as dimensões, o que suscita a hipótese de que estratégias construtivas e destrutivas e padrões de interação positivos e negativos podem coexistir dentro de um relacionamento conjugal.

Estes achados confirmam a compreensão de outros autores (Bertoni \& Bodenmann, 2010; Féres-Carneiro, 1998) de que os conflitos conjugais ocorrem, naturalmente, como fruto da interação de pessoas que almejam construir um projeto de vida em conjunto e, para tanto, precisarão discutir e negociar sobre pontos de vista divergentes e tentar chegar a um acordo. Consequentemente, adiando a resolução dos problemas pode haver um acúmulo de impasses que eclodirão com mais força e carga negativa do que se fossem resolvidos no contexto em que emergiram (Zordan, Wagner, \& Mosmann, 2012).

Nesse sentido, as estratégias de resolução dos conflitos conjugais são muito importantes. Possuem um caráter interacional e são definidas na literatura como os comportamentos dirigidos ao cônjuge na tentativa de gerenciar o problema e dar um desfecho para a situação. Idealmente, o conflito deve ser identificado com clareza pelos cônjuges, a fim de que estes possam dedicar-se ativamente à sua resolução (Carlson \& Dinkmeyer, 1987; Fincham, 2009; Garcia \& Tassara, 2001).

As estratégias de resolução de conflito mais efetivas envolvem controle emocional, orientação para a solução, confronto não ostensivo (Wheeler, Updegraff, \& Thayer, 2010), comunicação respeitosa, capacidade de escuta mútua e percepção do casamento como uma vivência que possui limitações (Hahlweg \& Richter, 2010; Tavora, 2009). As estratégias de resolução menos eficazes estão associadas aos comportamentos de racionalização excessiva e de negação, à percepção negativa do parceiro e da relação (Whiting, 2008) e ao pessimismo frente à resolução dos problemas conjugais (Rasera \& Guanaes, 2010).

Além disso, utilizar estratégias construtivas reverbera positivamente no relacionamento ao longo do tempo (Sierau \& Herzberg, 2012) e minimiza a possibilidade de separação e divórcio entre os casais que se propõem a desenvolver, por exemplo, habilidades comunicacionais como falar e ouvir (Hahlweg \& Richter, 2010). Segundo Anderson e Johnson (2010), o desenvolvimento de um ambiente mais positivo, em que os problemas são resolvidos via estratégias construtivas, reforça a aliança entre os cônjuges e os fortalece diante das situações de estresse no dia a dia.

De acordo com os estudos encontrados, percebe-se escassez de pesquisas sobre os motivos dos conflitos conjugais e sobre as estratégias de resolução no contexto nacional (Bolze et al., 2011, 2013). Na literatura internacional, existe significativa produção científica em torno destes temas, de modo que os pesquisadores dedicam-se ao estudo de variáveis específicas, envolvendo os conflitos conjugais e as formas de resolvê-los (Anderson \& Johnson, 2010; Bertoni \& Bodenmann, 2010; Dush \& Taylor, 2012; Graber, Laurenceau, Miga, Chango, \& Coan, 2011; Sierau \& Herzberg, 2012; Wheeler et al., 
2010; Whiting, 2008). Entretanto, por decisões teóricas e metodológicas, os pesquisadores utilizam-se de nomenclaturas distintas para estratégias equivalentes. A heterogeneidade no emprego dos termos interfere na compreensão das estratégias de resolução e das reverberações que provocam na conjugalidade.

Estudos internacionais investigam, há mais de vinte anos, os conflitos conjugais e as suas repercussões na vida dos cônjuges e no ambiente familiar. Tal fato aponta a importância de mapear e sistematizar os resultados de pesquisas, no contexto nacional e internacional e de indicar alternativas de investigação futura de um fenômeno inserido em uma conjuntura social e cultural idiossincrática (Benetti, 2006). Diante dessas considerações, o objetivo deste estudo foi analisar a literatura nacional e internacional sobre os conflitos conjugais e as estratégias de resolução empregadas pelos cônjuges.

\section{Método}

\section{Delineamento}

O presente estudo é uma revisão sistemática qualitativa. As revisões sistemáticas empregam análise crítica e síntese de estudos sobre determinada temática por meio de procedimentos sistemáticos, explícitos e replicáveis, e cujo objetivo principal é integrar os estudos, apontar divergências e convergências e orientar novas investigações (Sampaio \& Mancini, 2007). A elaboração desta revisão, com base nos autores citados, foi realizada através das etapas: (a) formulação da questão de pesquisa; (b) considerações acerca do estado da arte do tema investigado; (c) definição de critérios de inclusão e exclusão; (d) análise das características e resultados dos estudos recuperados; (e) apresentação dos resultados e discussão.

\section{Procedimentos de Busca}

A pesquisa foi realizada em dez bases de dados: Academic Search Complete, Biblioteca Cochrane, Ibecs (Indice Bibliográfico Español de Ciencias de la Salud), Index Psi, Lilacs (Literatura Latino-Americana e do Caribe em Ciências da Saúde), Medline (Medical Literature Analysis and Retrieval System Online), Portal de Evidências, PsycINFO, SciELO (Scientific Electronic Library Online) e Web of Science. Empregou-se descritores das ciências da saúde listados na Biblioteca Virtual em Saúde Decs/ BVS e o operador boleano AND para refinar os resultados da primeira etapa da busca. Os descritores e os termos equivalentes em inglês foram: (a) conflito conjugal $A N D$ casamento - marital conflict AND marriage e (b) resolução de problemas $A N D$ casamento - problem solving AND marriage.

Os critérios de inclusão marcados nos campos de busca das bases de dados foram: unitermos e suas combinações presentes nas palavras-chave, artigo científico de pesquisa empírica da área da psicologia, sem restrição de idioma ou país, disponível em texto completo nas bases entre janeiro de 2004 e dezembro de 2013, período delimitado devido ao número expressivo de pesquisas, especialmente na literatura internacional. A especificidade temática - estudos que investigaram os conflitos conjugais e estratégias de resolução, foi avaliada inicialmente através da leitura dos resumos. Foram critérios de exclusão: pesquisas apresentadas através de capítulo, livro, dissertação de mestrado ou tese de doutorado, estudos disponíveis apenas enquanto resumo, publicações que não passaram pelo critério de avaliação por pares, ano de publicação anterior a 2004 ou posterior a 2013, áreas de estudo que não a psicologia e artigos cujo foco não se restringia ao conflito conjugal e as estratégias de resolução, mas sobre associações e repercussões destes em outras variáveis pessoais, familiares e contextuais.

\section{Análise dos Dados}

Os artigos recuperados foram analisados de forma qualitativa por meio de procedimentos de análise de conteúdo (Bauer, 2008), quais sejam:

1. Leitura flutuante, exploração e pré-análise dos artigos. Nesta etapa foi construída uma tabela contendo características do estudo, seus objetivos e resultados;

2. Identificação e descrição dos resultados que responderam aos objetivos da pesquisa;

3. Identificação de unidades de significado e agrupamento destas por semelhança; 
4. Elaboração de eixos temáticos a partir dos agrupamentos formados. A elaboração dos eixos temáticos considerou a priori resultados associados aos motivos dos conflitos conjugais e às diferentes estratégias, construtivas e destrutivas, de resolução dos desacordos conjugais.

\section{Resultados}

Por meio dos descritores e critérios operacionais delimitados nesta revisão sistemática foram recuperados 567 artigos, conforme Figura 1: Academic Search Complete $(n=101)$, Biblioteca Cochrane $(n=21)$, Ibecs $(n=1)$, Index Psi $(n=17)$, Lilacs $(n=7)$, Medline $(n=113)$, Portal de Evidências $(n=1)$, PsycINFO $(n=35)$, SciELO $(n=8)$ e Web of Science $(\mathrm{n}=263)$. Destes estudos recuperados $(\mathrm{N}=567)$, foram excluídos 426 que investigavam diferentes variáveis associadas aos conflitos conjugais e às estratégias de resolução, quais sejam: consequências na vida dos filhos em diferentes etapas do desenvolvimento - crianças, adolescentes e jovens, questões de parentalidade, de coparentalidade e de família de origem, saúde física e mental como câncer, problemas respiratórios, depressão e, outros, relacionados ao consumo de álcool, à ansiedade, aos cuidados com a saúde, aos contextos de risco e à religião. Foram excluídos 97 artigos duplicados nas bases de dados, quatro pesquisas para validação de instrumentos sobre conflitos conjugais e estratégias de resolução, três artigos indisponíveis na versão completa nas bases pesquisadas, dois estudos de caso, dois artigos teóricos, duas pesquisas com intervenção e um artigo de revisão sistemática da literatura nacional. Desse modo, 30 artigos que investigaram especificamente os conflitos conjugais e as estratégias de resolução no casamento constituem esta revisão sistemática.

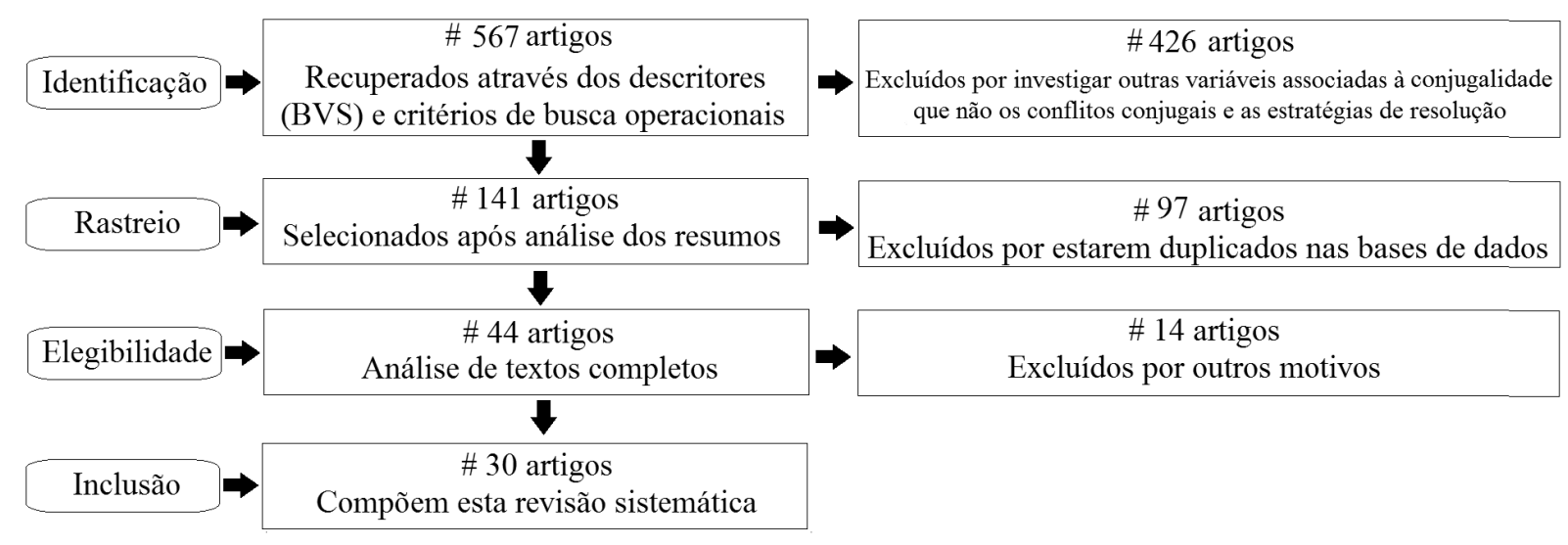

Figura 1. Fluxograma.

Percebe-se, conforme Tabela 1, que entre 2004 e 2013, foi publicado pelo menos um estudo ao ano envolvendo a temática dos conflitos conjugais ou das estratégias de resolução, com destaque para 2010 e 2012, em que houve o maior número de publicações $(n=5)$. Quanto ao delineamento foram encontrados estudos descritivos $(n=1)$, comparativos $(n=7)$, correlacionais $(n=7)$, longitudinais $(n=8)$ e explicativos $(n=24)$. Os países de realização dos estudos foram: Estados Unidos $(n=22)$; Estados Unidos e País de Gales $(n=1)$; Estados Unidos e Canadá $(n=1)$; Bélgica, Turquia, Itália, Taiwan, Brasil e Bolívia, todos com um estudo.
Através da análise de conteúdo, à qual os resultados dos estudos foram submetidos, originaram-se três eixos temáticos, conforme Tabela 2. No eixo I "Conflitos Conjugais" são apresentadas as categorias: (a) Compreensão consensual sobre os conflitos conjugais e (b) Motivos dos conflitos.

As pesquisas apontam que os desacordos conjugais são inevitáveis, ameaçam a satisfação e a qualidade conjugal ao longo do tempo se intensos e frequentes e que precisam ser gerenciados de alguma forma pelos parceiros ao invés de ignorados ou de a sua resolução ser protelada (Fincham et al., 2007; Mosmann \& Falcke, 
Tabela 1

Caracterização dos Estudos

\begin{tabular}{|c|c|c|c|c|}
\hline Estudos/Autores & Ano & Delineamento & Amostra & País \\
\hline Fincham, Beach, \& Davila & 2004 & Correlacional e Explicativo & 148 casais & $\begin{array}{l}\text { EUA e País } \\
\text { de Gales }\end{array}$ \\
\hline Tallman \& Hsiao & 2004 & $\begin{array}{l}\text { Longitudinal, Comparativo } \\
\text { e Explicativo }\end{array}$ & 574 casais & EUA \\
\hline Miller \& Rempel & 2004 & Explicativo & 75 casais & EUA \\
\hline $\begin{array}{l}\text { Verhofstadt, Buysse, Ickes, } \\
\text { De Clercq, \& Peene }\end{array}$ & 2005 & Comparativo & 53 casais & EUA \\
\hline $\begin{array}{l}\text { Verhofstadt, Buysse, } \\
\text { De Clercq, \& Goodwin }\end{array}$ & 2005 & Explicativo & 118 casais & Bélgica \\
\hline Johnson et al. & 2005 & $\begin{array}{c}\text { Comparativo, Correlacional } \\
\text { e Explicativo }\end{array}$ & 172 casais & EUA \\
\hline Simmons, Gordon, \& Chambless & 2005 & Correlacional & 59 casais & EUA \\
\hline Sanford & 2006 & Explicativo & 77 casais & EUA \\
\hline Bermúdez, Reyes, \& Wampler & 2006 & Comparativo & 191 casais & EUA \\
\hline Papp, Goeke-Morey, \& Cummings & 2007 & Comparativo e Explicativo & 100 casais & EUA \\
\hline Fincham, Beach, \& Davila & 2007 & $\begin{array}{l}\text { Longitudinal, Correlacional } \\
\text { e Explicativo }\end{array}$ & 96 casais & EUA \\
\hline McNulty, O’Mara, \& Karney & 2008 & Longitudinal e Explicativo & 251 casais & EUA \\
\hline Smith et al. & 2009 & Comparativo & 300 casais & EUA \\
\hline Karahan & 2009 & Longitudinal e Explicativo & 122 casais & Turquia \\
\hline $\begin{array}{c}\text { Seider, Hirschberger, Nelson, \& } \\
\text { Levenson }\end{array}$ & 2009 & Explicativo & 154 casais & EUA \\
\hline Paleari, Regalia, \& Fincham & 2010 & Explicativo & 92 casais & Itália \\
\hline Cheng & 2010 & Correlacional e Comparativo & 201 casais & Taiwan \\
\hline $\begin{array}{c}\text { Sullivan, Pasch, Johnson, \& } \\
\text { Bradbury }\end{array}$ & 2010 & Longitudinal e Explicativo & 172 casais & EUA \\
\hline $\begin{array}{l}\text { Markman, Rhoades, Stanley, } \\
\text { Ragan, \& Whitton }\end{array}$ & 2010 & Longitudinal e Explicativo & 208 casais & EUA \\
\hline McNulty \& Russell & 2010 & Explicativo & 207 casais & EUA \\
\hline Mosmann \& Falcke & 2011 & Descritivo & 149 casais & Brasil \\
\hline Madhyastha, Hamaker, \& Gottman & 2011 & Explicativo & 254 casais & EUA \\
\hline Rehman et al. & 2011 & Explicativo & 15 casais & EUA e Canadá \\
\hline Hoppmann \& Blanchard-Fields & 2011 & Explicativo & 49 casais & EUA \\
\hline $\begin{array}{l}\text { Leggett, Roberts-Pittman, } \\
\text { Byczek, \& Morse }\end{array}$ & 2012 & Correlacional e Explicativo & 977 indivíduos & EUA \\
\hline Cundiff, Smith, \& Frandsen & 2012 & Correlacional e Explicativo & 300 casais & EUA \\
\hline $\begin{array}{c}\text { Stieglitz, Gurven, Kaplan, } \\
\text { \& Winking }\end{array}$ & 2012 & Explicativo & 46 indivíduos & Bolívia \\
\hline Schoebi, Karney, \& Bradbury & 2012 & Longitudinal e Explicativo & 172 casais & EUA \\
\hline Uysal, Lin, Knee, \& Bush & 2012 & Explicativo & 265 indivíduos & EUA \\
\hline $\begin{array}{l}\text { Veldorale-Brogan, Lambert, } \\
\text { Fincham, \& DeWall }\end{array}$ & 2013 & Longitudinal e Explicativo & 412 indivíduos & EUA \\
\hline
\end{tabular}


2011; Tallman \& Hsiao, 2004). Na categoria "Motivos dos conflitos", dois estudos abordaram a questão, indicando que os impasses estão relacionados à educação dos filhos, à negociação sobre o tempo que os parceiros passam juntos, à administração do dinheiro, à divisão das tarefas domésticas (Mosmann \& Falcke, 2011) e por questões de infidelidade e dinheiro (Stieglitz et al., 2012).

No eixo II "Estratégias de resolução dos conflitos conjugais" são apresentadas as categorias: (a) Estratégias de resolução de conflito construtivas e (b) Estratégias de resolução de conflito destrutivas. Na resolução construtiva, as estratégias encontradas foram: benevolência, cooperação, suporte, validação, autocontrole, comunicação positiva, utilização de pronomes de integração (nós), identificação e abordagem ativa dos problemas, inclinação à mudança de comportamento, bom humor e demonstração de interesse em resolver os desacordos. $\mathrm{Na}$ resolução destrutiva, as estratégias encontradas foram: retaliação, autoproteção, ataque, utilização de pronomes de separação (tu), abordagem passiva do problema, comunicação negativa, ocultação de informações do parceiro e esquiva (Bermúdez et al., 2006; Fincham et al., 2004, 2007; Hoppmann \& Blanchard-Fields, 2011; Johnson et al., 2005; Leggett et al., 2012; Markman et al., 2010; McNulty et al., 2008; McNulty \& Russell, 2010; Paleari et al., 2010; Sanford, 2006; Schoebi et al., 2012; Seider et al., 2009; Simmons et al., 2005; Sullivan et al., 2010; Tallman \& Hsiao, 2004; Uysal et al., 2012; Veldorale-Brogan et al., 2013; Verhofstadt, Buysse, Ickes, et al., 2005).

No eixo III "Variáveis associadas aos conflitos conjugais e às estratégias de resolução" são apresentadas as categorias: (a) Sexo, (b) Características individuais, (c) Processos interacionais e (d) Tempo de união. Os estudos apontam que homens e mulheres têm atitudes e utilizam estratégias diferentes durante as situações de conflito, emergindo a categoria "Sexo". Homens têm, predominantemente, comportamentos de retirada, inflexão, esquiva, autoritarismo, crítica excessiva e culpabilização das esposas, escuta superficial, seletiva, defensiva e armada e dificuldades para expressar emoções. Mulheres têm mais comportamentos de demanda e auto revelação, atribuição de maior gravidade ao problema, mudanças acentuadas nos níveis de satisfação com o relacionamento, sentem e expressam emoções intensas com xingamento, mau humor e interrupção da comunicação (Cheng, 2010; Karahan, 2009; McNulty et al., 2008; Paleari et al., 2010; Papp et al., 2007; Rehman et al., 2011; Smith et al., 2009; Uysal et al., 2012; Verhofstadt, Buysse, De Clercq, et al., 2005).

As pesquisas indicam que as "Características individuais" dos cônjuges interferem na resolução dos conflitos conjugais em três momentos: antes do conflito ocorrer, quanto à expectativa positiva ou negativa sobre si, em relação ao parceiro e sobre o desfecho da situação, durante e após o conflito. Além disso, a empatia, a percepção de competência em si mesmo e no cônjuge, a atribuição de gravidade ao problema com base nas concepções de mundo pessoais e a interpretação dos motivos subjacentes ao conflito, são centrais à resolução dos problemas na conjugalidade (Cundiff et al., 2012; Hoppmann \& Blanchard-Fields, 2011; Leggett et al., 2012; McNulty et al., 2008; Miller \& Rempel, 2004; Paleari et al., 2010; Sanford, 2006; Tallman \& Hsiao, 2004; Veldorale-Brogan et al., 2013).

Em "Processos interacionais" são discutidas a influência mútua de positividade e negatividade entre os cônjuges e os padrões de interação conjugal. Os resultados dos estudos apontam que por meio das características de cada cônjuge se estabelece um contexto relacional único do par conjugal em que ambos estão implicados e pelo qual são corresponsáveis (Cundiff et al., 2012; Johnson et al., 2005; Madhyastha et al., 2011).

Na categoria sobre o "Tempo de união", as pesquisas encontradas apontam que parceiros em casamentos de longa duração, mais de vinte anos, apresentam menores níveis de ansiedade, de submissão, de negatividade, têm maiores níveis de controle e de positividade e usam estratégias de colaboração durante os conflitos conjugais, quando comparados aos casais mais jovens, casados a menos de cinco anos (Hoppmann \& Blanchard-Fields, 2011; Smith et al., 2009; Stieglitz et al., 2012). 
Tabela 2

Eixos, Categorias e Resultados dos Estudos

\begin{tabular}{|c|c|c|c|}
\hline $\begin{array}{l}\text { Eixos } \\
\text { Temáticos }\end{array}$ & Categorias & Resultados & Estudos \\
\hline \multirow{3}{*}{$\begin{array}{l}\text { Conflito } \\
\text { conjugal }\end{array}$} & $\begin{array}{l}\text { Compreensão } \\
\text { consensual } \\
\text { acerca dos } \\
\text { conflitos } \\
\text { conjugais }\end{array}$ & $\begin{array}{l}\text { Inevitáveis; ameaçam a satisfação e a } \\
\text { qualidade conjugal se intensos e frequentes; } \\
\text { precisam ser tratados }\end{array}$ & $\begin{array}{l}\text { Fincham et al., 2007; Mosmann \& } \\
\text { Falcke, 2011; Tallman \& Hsiao, } 2004\end{array}$ \\
\hline & \multirow{2}{*}{$\begin{array}{l}\text { Motivos } \\
\text { de conflito }\end{array}$} & $\begin{array}{l}\text { Filhos; tempo que os parceiros passam } \\
\text { juntos; dinheiro; tarefas domésticas }\end{array}$ & Mosmann \& Falcke, 2011 \\
\hline & & Infidelidade; dinheiro & Stieglitz et al., 2012 \\
\hline \multirow[t]{2}{*}{$\begin{array}{l}\text { Estratégias } \\
\text { de resolução } \\
\text { de conflito }\end{array}$} & $\begin{array}{l}\text { Estratégias } \\
\text { de resolução } \\
\text { construtivas }\end{array}$ & $\begin{array}{c}\text { Benevolência; cooperação; suporte; } \\
\text { validação; autocontrole; comunicação } \\
\text { positiva; utilização de pronomes de } \\
\text { integração - nós; identificação e abordagem } \\
\text { ativa dos problemas; inclinação à mudança } \\
\text { de comportamento; bom humor, } \\
\text { entusiasmo e demonstração de interesse } \\
\text { resolver os conflitos; }\end{array}$ & $\begin{array}{c}\text { Bermúdez et al., 2006; } \\
\text { Fincham et al., 2004, 2007; } \\
\text { Hoppmann \& Blanchard-Fields, } \\
\text { 2011; Johnson et al., 2005; } \\
\text { Leggett et al., 2012; Markman et al., } \\
\text { 2010; McNulty et al., 2008; McNulty } \\
\text { \& Russell, 2010; Paleari et al., 2010; } \\
\text { Sanford, 2006; Schoebi et al., 2012; }\end{array}$ \\
\hline & $\begin{array}{l}\text { Estratégias } \\
\text { de resolução } \\
\text { destrutivas }\end{array}$ & $\begin{array}{c}\text { Retaliação; autoproteção; ataque; utilização } \\
\text { de pronomes de separação - tu; abordagem } \\
\text { passiva do problema; comunicação negativa; } \\
\text { ocultação de informações do parceiro; } \\
\text { esquiva }\end{array}$ & $\begin{array}{l}\text { Seider et al., 2009; Simmons et al., } \\
\text { 2005; Sullivan et al., 2010; Tallman } \\
\text { \& Hsiao, 2004; Uysal et al., 2012; } \\
\text { Veldorale-Brogan et al., 2013; } \\
\text { Verhofstadt, Buysse, Ickes, et al., } 2005\end{array}$ \\
\hline
\end{tabular}

Homens: comportamentos de retirada, rigidez, evitação, autoritarismo, crítica e culpabilização das esposas; escuta superficial, seletiva, defensiva e armada, dificuldades para expressar emoções

Variáveis associadas aos conflitos conjugais e às estratégias de resolução
Características individuais

Fator diádico
Sexo

Mulheres: comportamentos de demanda e auto revelação, atribuição de maior gravidade ao problema, mudanças acentuadas nos níveis de satisfação com a relação, experimental e expressam emoções intensas através de xingamento, mau humor e interrupção da comunicação

Expectativa positiva ou negativa de si, do parceiro e sobre o desfecho da situação; empatia, percepção de competência em si e no cônjuge, atribuição de gravidade ao problema; interpretação que se faz dos motivos subjacentes ao conflito

Influência mútua de positividade e negatividade durante a interação; padrões de interação conjugal

Casais mais velhos apresentam menores níveis de ansiedade, submissão, negatividade e uso

Tempo de união de estratégias diretas; têm maiores níveis de controle, positividade e uso de estratégias de colaboração quando comparados aos casais mais jovens, casados há menos de cinco anos
Cheng, 2010; Karahan, 2009;

McNulty et al., 2008;

Paleari et al., 2010;

Papp et al., 2007;

Rehman et al., 2011;

Smith et al., 2009;

Uysal et al., 2012;

Verhofstadt, Buysse, De Clercq, et al., 2005

Cundiff et al., 2012;

Hoppmann \& Blanchard-Fields,

2011; Leggett et al., 2012;

McNulty et al., 2008; Miller \& Rempel, 2004; Paleari et al., 2010; Sanford, 2006; Tallman \& Hsiao, 2004; Veldorale-Brogan et al., 2013

Cundiff et al., 2012;

Johnson et al., 2005;

Madhyastha et al., 2011

Hoppmann \& Blanchard-Fields, 2011; Smith et al., 2009; Stieglitz et al., 2012 


\section{Discussão}

Os anos de publicação dos estudos revisados revelam que a temática está sendo investigada no meio científico, embora não se possa afirmar que houve aumento no número de publicações, apenas que a produção se concentrou em período mais recente, 2010 e 2012. Uma característica interessante desses estudos refere-se aos delineamentos empregados. A maior parte das investigações utilizou delineamento longitudinal e explicativo, o que sugere uma preocupação dos pesquisadores em qualificar suas pesquisas por meio de delineamentos mais sofisticados do ponto de vista metodológico, visando aperfeiçoar os estudos e avançar no conhecimento científico sobre a temática investigada.

Quanto aos países de realização destas pesquisas, os Estados Unidos se destacam expressivamente em relação aos demais. Esse dado indica, inevitavelmente, que os resultados dos estudos fornecerão informações associadas à realidade norte americana e às idiossincrasias próprias dessa cultura e, portanto, que generalizações e associações a outros contextos devem ser feitas com cautela. Além disso, esses achados indicam a carência de estudos no contexto brasileiro, onde foi encontrado apenas um artigo.

Na primeira categoria do Eixo I, intitulada "Compreensão consensual sobre os conflitos conjugais" identificou-se que os atritos entre os cônjuges são inevitáveis e fazem parte da conjugalidade, mas necessitam de resolução pois há o risco de ameaçar a satisfação e a qualidade conjugal e aumentar em intensidade e frequência ao longo do tempo de casamento. Este achado pode indicar que o acúmulo de situações pendentes, dentro de um relacionamento, torna cada vez mais difícil a sua resolução, repercute negativamente nos parceiros e complexifica o problema que será associado a outros eventos não resolvidos no passado. Pode-se perder o foco do real motivo de conflito, tornando-o maior do que realmente é e, gerando repercussões emocionais como mágoa, raiva e desinteresse pelo relacionamento.

Quanto aos "Motivos dos conflitos", emergiu um número escasso de pesquisas. No estudo de Mosmann e Falcke (2011) foram apontados os desacordos entre os parceiros quanto à educação dos filhos, ao tempo que passam juntos, ao dinheiro e às tarefas domésticas. No estudo de Stieglitz et al. (2012) foram encontrados o dinheiro e a infidelidade como motivo de conflito. Nas duas pesquisas o dinheiro foi tema gerador de discórdia, talvez porque as finanças de um casal sejam mais difíceis de gerir do que outras questões. Um entendimento para este resultado é que os casais aprenderam a nominar de dificuldades financeiras seus principais conflitos relacionais, sem conseguir distinguir questões subjacentes como problemas de comunicação, por exemplo. Podem ter encontrado na questão financeira, terreno fértil para dar um sentido palpável à outras angústias e desencontros conjugais.

No eixo II "Estratégias de resolução dos conflitos conjugais" são apresentadas as categorias: (a) Estratégias de resolução de conflito construtivas e (b) Estratégias de resolução de conflito destrutivas. As estratégias encontradas nos artigos revisados (Hahlweg \& Richter, 2010; Rasera \& Guanaes, 2010; Tavora, 2009; Wheeler et al., 2010; Whiting, 2008) se equiparam, quase que em sua totalidade, e constituem um corpus de conhecimento significativo sobre a temática, especialmente, na literatura internacional.

Por meio deste resultado, identifica-se que as estratégias de resolução construtivas apontam a importância de postura mais flexível e tolerante diante dos problemas conjugais e limitações do cônjuge, da percepção otimista dos conflitos e de assumir que a sua ocorrência e resolução envolvem responsabilidade mútua. As estratégias que reverberam destrutivamente na conjugalidade estão relacionadas aos comportamentos individualizados frente às questões conjugais $\mathrm{e}$ às dificuldades em flexibilizar pensamentos e se conectar com o que a relação e o parceiro têm de positivo. Remetem a um sentido de competição em que há dois objetivos: atacar ou se proteger. É preciso comprovar que o outro é o responsável pelos problemas ou se defender das possíveis acusações que ele(a) fará. Quando o princípio da estratégia pressupõe a competição, cria a expectativa nos cônjuges de que haverá somente um vencedor da disputa afetiva. Tal crença retroa- 
limenta o investimento constante de ataque e de proteção de ambos os cônjuges. Eles acreditam que o problema da relação conjugal é o comportamento do parceiro e não percebem o quanto cada um contribui para a relação continuar sendo motivo de sofrimento e de investimento nocivo para a dinâmica relacional conjugal.

No eixo III "Variáveis associadas aos conflitos conjugais e às estratégias de resolução" são apresentadas as categorias: (a) Sexo, (b) Características individuais, (c) Processos interacionais e (d) Tempo de união. Na categoria, "Sexo", estudos apontam diferenças significativas entre homens e mulheres quanto à percepção dos conflitos conjugais e das estratégias de resolução adotadas. Os resultados podem indicar que as dificuldades para resolver os conflitos estão associadas às diferentes percepções, expectativas e comportamentos que homens e mulheres têm diante dos problemas conjugais, das relações amorosas e do jeito de expressar tais questões, corroborando o que apontam outras pesquisas (Dush \& Taylor, 2012; Garcia \& Tassara, 2001; Giddens, 1993).

Refletir dessa forma é reconhecer mais um elemento que interfere na tentativa construtiva ou destrutiva de resolver os desacordos conjugais. As diferenças entre maridos e esposas dentro do casamento têm como consequência, segundo os resultados das pesquisas, distintos métodos de resolver os conflitos e de reagir frente a eles. Essas diferenças impactam nos níveis de satisfação com a relação por estarem associadas, teoricamente, ao investimento emocional feminino, à dificuldade masculina de lidar com os sentimentos e à forma como ambos os cônjuges tendem a tratar os assuntos conjugais.

$\mathrm{Na}$ categoria sobre as "Características individuais" os estudos apontam que o processo pessoal de cada cônjuge interfere diretamente na resolução dos problemas conjugais antes, durante e após o conflito ocorrer. Esses resultados provocam reflexões quanto à perspectiva relacional do conflito e fazem emergir a hipótese de que os desacordos estariam associados, mais fortemente, às características individuais (Silva \& Vandenberghe, 2009) do que aos processos interacionais.
Nesse entendimento, os parceiros trazem preconcepções que funcionariam como profecias autorrealizadoras do desfecho do conflito (Sanford, 2006), remetendo para processos anteriores à constituição do vínculo conjugal. Esses resultados podem indicar que o relacionamento marital é influenciado, mais fortemente, pelo reflexo do micro nível do conflito conjugal no indivíduo, efeito ator e, também, pela inércia emocional, dificuldade de mudar e/ou flexibilizar pensamentos e comportamentos, característicos do funcionamento de cada cônjuge, desde os anos iniciais do casamento e, até mesmo, anteriores a constituição do vínculo amoroso.

Por outro lado, na categoria "Processos interacionais" foram encontrados dois estudos sobre a influência mútua de positividade e negatividade que cada parceiro traz para a interação (Cundiff et al., 2012; Madhyastha et al., 2011). Compreende-se, por meio desses resultados, que o par conjugal, ao decidir pela convivência, constitui um universo intersubjetivo que se mantém em constante atualização e que exigirá dos cônjuges postura para tomar decisões que, a partir da união matrimonial, favorecerão a conjugalidade e não apenas as individualidades.

Os cônjuges precisam ter consciência de que pertencem a um contexto sócio-econômico-cultural-espiritual único, mas que torna-se compartilhado ao se casar. Durante o exercício da conjugalidade e da vivência das diferentes etapas do ciclo de vida conjugal emergirão demandas em que devem estar contemplados valores, desejos e necessidades individuais e conjugais. Para que isso aconteça o diálogo e a flexibilidade são necessários ao processo de tomada de decisão que só poderá ser nominada, sentida e vivida por aquele par marital. O casal estabelece um padrão de funcionamento que guia suas decisões no transcorrer do casamento em que ambos partilham, retroalimentam e reproduzem dinâmicas relacionais aprendidas na sua vivência com as famílias de origem.

O "Tempo de união" emergiu como uma variável que provoca alterações na dinâmica conjugal. Os estudos apontaram diferenças com relação ao comportamento e aos estilos de resolução dos conflitos entre casamentos de longa duração 
e casamentos de curta duração. Esse achado pode indicar que, com o passar do tempo de união, acontece um processo de amadurecimento natural das percepções, necessidades, expectativas e comportamentos diante dos conflitos conjugais. As estratégias estariam sujeitas à interferência do momento do ciclo vital individual, conjugal e familiar dos parceiros.

Nos primeiros anos de casamento, os conflitos podem ser mais intensos, frequentes e dificeis de resolver por ser um período de adaptação entre os cônjuges. $\mathrm{O}$ casal precisará gerenciar demandas cotidianas como, divisão das tarefas domésticas, partilha dos gastos, alinhamento dos projetos de vida pessoal, profissional e conjugal, nascimento de filhos, entre outros. Tal período, demandaria maior esforço, investimento e apoio entre os parceiros. Passada a etapa inicial do casamento é possível que os cônjuges compreendam que a vida conjugal e familiar exige investimento mútuo e constante e que certas situações não serão resolvidas dentro das suas expectativas. Perceberiam que as diferenças e os desacordos conjugais são naturais, importantes para o desenvolvimento da conjugalidade e sempre existirão, apesar de gerar, muitas vezes, desconforto, mágoa e culminar em separação.

\section{Considerações Finais}

Por meio dos estudos revisados, identificou-se que os motivos dos conflitos não têm sido foco expressivo de investigação, possivelmente, por se compreender que são fatores secundários à resolução dos problemas. Nessa perspectiva, variáveis como sexo, características individuais, tempo de união e processos interacionais estiveram associadas às situações de conflito e às estratégias de resolução.

Quanto à variável tempo de união, analisar as diferentes fases do ciclo vital conjugal pode ser um tema de investigação relevante e oportuno. A avaliação dessa variável pode fornecer indicativos de como planejar intervenções que possam prevenir certas expressões de conflito e dificuldades no início do relacionamento amoroso.

Percebe-se, através destes resultados, que os conflitos conjugais e as estratégias de resolução não encerram em si a compreensão das dinâmicas conjugais. A inter-relação entre as características pessoais dos cônjuges e a qualidade do processo interacional, influenciado por questões individuais e pelo movimento antecipado ou tardio de resolução dos conflitos, culminará em um ambiente conjugal mais positivo ou mais negativo. O desenvolvimento de modelos explicativos poderia integrar as variáveis encontradas e apontar outras possíveis compreensões sobre a temática investigada.

Por decisões metodológicas, não foi possível discutir os dados das pesquisas de forma quantitativa. Estudos de revisão sistemática podem analisar os artigos encontrados noutra perspectiva, propondo diferentes discussões e reflexões sobre os conflitos conjugais e as estratégias de resolução. Ressalta-se que apenas um estudo nacional compõe esta revisão, indicando a necessidade premente de pesquisas dentro desse contexto, para que clínicos e outros pesquisadores tenham evidências empíricas que subsidiem a teoria e a prática profissional com base em resultados ajustados às idiossincrasias da cultura brasileira.

\section{Referências}

Anderson, S. R., \& Johnson, L. N. (2010). A dyadic analysis of the between and within system alliances on distress. Family Process, 49(2), 220-235. doi:10.1111/j.1545-5300.2010.01319.x

Bauer, M. W. (2008). Análise de conteúdo clássica: Uma revisão. In M. W. Bauer \& G. Gaskell, Pesquisa qualitativa com texto, imagem e som: Um manual prático (P. A. Guareschi, Trad., 7. ed., pp. 189-217). Petrópolis, RJ: Vozes.

Benetti, S. P. C. (2006). Conflito conjugal: Impacto no desenvolvimento psicológico da criança e do adolescente. Psicologia: Reflexão e Crítica, 19(2), 261-268. Recuperado em http://www. scientificcircle.com/pt/journal/103/psicol-reflex-crit/2006/0/19/2/

Bermúdez, J. M., Reyes, N. A., \& Wampler, K. S. (2006). Conflict resolution styles among Latino couples. Journal of Couple \& Relationship Therapy, 5(4), 1-21. doi:10.1300/J398v05n04_01

Bertoni, A., \& Bodenmann, G. (2010). Satisfied and dissatisfied couples: Positive and negative di- 
mensions, conflict styles, and relationships with family of origin. European Psychologist, 15(3), 175-184. doi:10.1027/1016-9040/a000015

Bolze, S. D. A., Schmidt, B., Crepaldi, M. A., \& Vieira, M. L. (2011). Conflito conjugal: Uma revisão da produção científica brasileira. Pensando Familias, 15(2), 51-69. Recuperado em http:// newpsi.bvs-psi.org.br/cgi-bin/wxis 1660.exe/ iah/

Bolze, S. D. A., Schmidt, B., Crepaldi, M. A., \& Vieira, M. L. (2013). Relacionamento conjugal e táticas de resolução de conflito entre casais. Actualidades en Psicología, 27(114), 71-85. Recuperado em http://kerwa.ucr.ac.cr/ handle $/ 10669 / 8806 /$ browse?type $=$ dateissued

Carlson, J., \& Dinkmeyer, D. (1987). Adlerian marriage therapy. The American Journal of Family Therapy, 15(4), 326-332. doi:10.1080/01926188708250692

Cheng, C. C. (2010). A study of inter-cultural marital conflict and satisfaction in Taiwan. International Journal of Intercultural Relations, 34(4), 354-362. doi:10.1016/j.ijintrel.2010.04.005

Cummings, E. M. (1998). Children exposed to marital conflict and violence: Conceptual and theoretical directions. In G. Holden, B. Geffner, \& E. Jouriles (Eds.), Children exposed to marital violence: Theory, research, and applied issues (pp. 21-53). Washington, DC: American Psychological Association.

Cundiff, J. M., Smith, T. W., \& Frandsen, C. A. (2012). Incremental validity of spouse ratings versus self-reports of personality as predictors of marital quality and behavior during marital conflict. Psychological Assessment, 24(3), 676684. doi:10.1037/a0026637

Dush, C. M. K., \& Taylor, M. G. (2012). Trajectories of marital conflict across the life course: Predictors and interactions with marital happiness trajectories. Journal of Family Issues, 33(3), 341368. doi:10.1177/0192513X11409684

Féres-Carneiro, T. (1998). Casamento contemporâneo: O difícil convívio da individualidade com a conjugalidade. Psicologia: Reflexão e Crítica, 11(2), 379-394. Recuperado em http://www.redalyc.org/articulo.oa?id=18811214

Fincham, F. D. (2009). Marital conflict. In Encyclopedia of Human Relationships: Vol. 1 (pp. 298 303). Thousand Oaks, CA: Sage.
Fincham, F. D., Beach, S. R., \& Davila, J. (2004). Forgiveness and conflict resolution in marriage. Journal of Family Psychology, 18(1), 72-81. doi:10.1037/0893-3200.18.1.72

Fincham, F. D., Beach, S. R., \& Davila, J. (2007). Longitudinal relations between forgiveness and conflict resolution in marriage. Journal of Family Psychology, 21(3), 542-545. doi:10.1037/08933200.21.3.542

Garcia, M. L. T., \& Tassara, E. T. O. (2001). Estratégias de enfrentamento do cotidiano conjugal. Psicologia: Reflexão e Crítica, 14(3), 635-642. Recuperado em http://www.scientificcircle.com/ pt/journal/103/psicol-reflex-crit/2001/0/14/3/

Giddens, A. (1993). A transformação da intimidade: Sexualidade, amor e erotismo nas sociedades modernas (M. Lopes, Trad.). São Paulo, SP: Universidade Estadual Paulista "Júlio de Mesquita Filho".

Gottman, J. M. (1998). Psychology and the study of marital processes. Annual Review of Psychology, 49(1), 169-197. doi:10.1146/annurev. psych.49.1.169

Graber, E. C., Laurenceau, J. P., Miga, E., Chango, J., \& Coan, J. (2011). Conflict and love: Predicting newlywed marital outcomes from two interaction contexts. Journal of Family Psychology, 25(4), 541-550. doi:10.1037/a0024507

Hahlweg, K., \& Richter, D. (2010). Prevention of marital instability and distress: Results of an 11year longitudinal follow-up study. Behaviour Research and Therapy, 48(5), 377-383. doi: 10.1016/j.brat.2009.12.010

Hoppmann, C. A., \& Blanchard-Fields, F. (2011). Problem-solving variability in older spouses: How is it linked to problem-, person-, and couple-characteristics? Psychology and Aging, 26(3), 525-531. doi:10.1037/a0024114

Johnson, M. D., Cohan, C. L., Davila, J., Lawrence, E., Rogge, R. D., Karney, B. R., ...Bradbury, T. N. (2005). Problem-solving skills and affective expressions as predictors of change in marital satisfaction. Journal of Consulting and Clinical Psychology, 73(1), 15-27. doi:10.1037/0022006X.73.1.15

Karahan, T. F. (2009). The effects of a couple communication program on the conflict resolution skills and active conflict tendencies of Turkish couples. Journal of Sex \& Marital Therapy, 35(3), 220-229. doi:10.1080/00926230802716344 
Leggett, D. G., Roberts-Pittman, B., Byczek, S., \& Morse, D. T. (2012). Cooperation, conflict, and marital satisfaction: Bridging theory, research, and practice. The Journal of Individual Psydiology, 68(2), 182-199.

Madhyastha, T. M., Hamaker, E. L., \& Gottman, J. M. (2011). Investigating spousal influence using moment-to-moment affect data from marital conflict. Journal of Family Psychology, 25(2), 292-300. doi:10.1037/a0023028

Markman, H. J., Rhoades, G. K., Stanley, S. M., Ragan, E. P., \& Whitton, S. W. (2010). The premarital communication roots of marital distress and divorce: The first five years of marriage. Journal of Family Psychology, 24(3), 289-298. doi:10.1037/a0019481

McNulty, J. K., O’Mara, E. M., \& Karney, B. R. (2008). Benevolent cognitions as a strategy of relationship maintenance: "Don't sweat the small stuff" ... but it is not all small stuff. Journal of Personality and Social Psychology, 94(4), 631-646. doi:10.1037/0022-3514.94.4.631

McNulty, J. K., \& Russell, V. M. (2010). When "negative" behaviors are positive: A contextual analysis of the long-term effects of problemsolving behaviors on changes in relationship satisfaction. Journal of Personality and Social Psychology, 98(4), 587-604. doi:10.1037/ a0017479

Miller, P. J., \& Rempel, J. K. (2004). Trust and partner-enhancing attributions in close relationships. Personality and Social Psychology Bulletin, 30(6), 695-705. doi:10.1177/0146167203262803

Mosmann, C., \& Falcke, D. (2011). Conflitos conjugais: Motivos e frequência. Revista da SPAGESP, 12(2), 5-16. Recuperado em http://pepsic.bvsalud.org/scielo.php?script $=$ sci_arttext\&p id $=$ S1677-29702011000200002

Paleari, F. G., Regalia, C., \& Fincham, F. D. (2010). Forgiveness and conflict resolution in close relationships: Within and cross partner effects. Universitas Psychologica, 9(1), 35-56. Retrieved from http://www.redalyc.org/articulo. oa?id $=64712156004$

Papp, L. M., Goeke-Morey, M. C., \& Cummings, E. M. (2007). Linkages between spouses psychological distress and marital conflict in the home. Journal of Family Psychology, 21(3), 533-537. Retrieved from http://psycnet.apa.org/journals/ fam $/ 21 / 3 / 533 /$
Rasera, E. F., \& Guanaes, C. (2010). Momentos marcantes na construção da mudança em terapia familiar. Psicologia Teoria e Pesquisa, 26(2), 315-322. Recuperado em https://revistaptp.unb. br/index.php/ptp/article/viewArticle/383

Rehman, U. S., Janssen, E., Newhouse, S., Heiman, J., Holtzworth-Munroe, A., Fallis, E., \& Rafaeli, E. (2011). Marital satisfaction and communication behaviors during sexual and nonsexual conflict discussions in newlywed couples: A pilot study. Journal of Sex \& Marital Therapy, 37(2), 94-103. doi:10.1080/0092623X.2011.547352

Sampaio, R. F., \& Mancini, M. C. (2007). Estudos de revisão sistemática: Um guia para síntese criteriosa da evidência científica. Revista Brasileira de Fisioterapia, 11(1), 83-89. doi:10.1590/ S1413-35552007000100013

Sanford, K. (2006). Communication during marital conflict: When couples alter their appraisal, they change their behavior. Journal of Family Psychology, 20(2), 256-265. doi:10.1037/08933200.20 .2 .256

Schoebi, D., Karney, B. R., \& Bradbury, T. N. (2012). Stability and change in the first 10 years of marriage: Does commitment confer benefits beyond the effects of satisfaction? Journal of Personality and Social Psychology, 102(4), 729742. doi:10.1037/a0026290

Seider, B. H., Hirschberger, G., Nelson, K. L., \& Levenson, R. W. (2009). We can work it out: Age differences in relational pronouns, physiology, and behavior in marital conflict. Psychology and Aging, 24(3), 604-613. doi:10.1037/a0016950

Sierau, S., \& Herzberg, P. Y. (2012). Conflict resolution as a dyadic mediator: Considering the partner perspective on conflict resolution. European Journal of Personality, 26(3), 221-232. doi:10.1002/per.828

Silva, L. P., \& Vandenberghe, L. (2009). Comunicação versus resolução de problemas numa sessão única de terapia comportamental de casal. Revista BTCC, 11(1), 43-60. Recuperado em http:// www.usp.br/rbtcc/index.php/RBTCC/article/ view/383

Simmons, R. A., Gordon, P. C., \& Chambless, D. L. (2005). Pronouns in marital interaction what do "you" and "i" say about marital health? Psychological Science, 16(12), 932-936. Retrieved from http://pss.sagepub.com/content/16/12/932. short 
Smith, T. W., Berg, C. A., Florsheim, P., Uchino, B. N., Pearce, G., Hawkins, M., ...Olsen-Cerny, C. (2009). Conflict and collaboration in middleaged and older couples: I age differences in agency and communion during marital interaction. Psychology and Aging, 24(2), 259-273. doi:10.1037/a0015609

Stieglitz, J., Gurven, M., Kaplan, H., \& Winking, J. (2012). Infidelity, jealousy, and wife abuse among Tsimane forager-farmers: Testing evolutionary hypotheses of marital conflict. Evolution and Human Behavior, 33(5), 438-448. doi:10.1016/j.evolhumbehav.2011.12.006

Sullivan, K. T., Pasch, L. A., Johnson, M. D., \& Bradbury, T. N. (2010). Social support, problem solving, and the longitudinal course of newlywed marriage. Journal of Personality and Social Psychology, 98(4), 631-644. doi:10.1037/ a0017578

Tallman, I., \& Hsiao, Y. L. (2004). Resources, cooperation, and problem solving in early marriage. Social Psychology Quarterly, 67(2), 172-188. doi:10.1177/019027250406700204

Tavora, M. T. (2009). Contrato emocional e código de ética: Pilares da reconstrução conjugal. Psico, 40(1), 50-57. Recuperado em http://revistaseletronicas.pucrs.br/ojs/index.php/revistapsico/ article/viewArticle/3999

Torossian, M. S., Heleno, M. G. V., \& Vizzotto, M. M. (2009). Relacionamento conjugal e o fenômeno da violência doméstica: Um estudo de caso. Psicologia da Saúde, 17(1), 12-16.

Uysal, A., Lin, H. L., Knee, C. R., \& Bush, A. L. (2012). The association between self-concealment from one's partner and relationship well-being. Personality and Social Psychology Bulletin, 38(1), 39-51. doi:10.1177/0146167211429331

Veldorale-Brogan, A., Lambert, N. M., Fincham, F. D., \& DeWall, C. N. (2013). The virtue of problem-solving: Perceived partner virtues as predictors of problem-solving efficacy. Personal Relationships, 20(3), 511-523. doi:10.1111/ j.1475-6811.2012.01421.x
Verhofstadt, L. L., Buysse, A., De Clercq, A., \& Goodwin, R. (2005). Emotional arousal and negative affect in marital conflict: The influence of gender, conflict structure, and demand-withdrawal. European Journal of Social Psychology, 35(4), 449-467. doi:10.1002/ejsp.262

Verhofstadt, L. L., Buysse, A., Ickes, W., De Clercq, A., \& Peene, O. J. (2005). Conflict and support interactions in marriage: An analysis of couples interactive behavior and on-line cognition. Personal Relationships, 12(1), 23-42. doi:10.1111/ j.1350-4126.2005.00100.x

Wheeler, L. A., Updegraff, K. A., \& Thayer, S. M. (2010). Conflict resolution in Mexican-origin couples: Culture, gender, and marital quality. Journal of Marriage and Family, 72(4), 9911005. doi:10.1111/j.1741-3737.2010.00744.x

Whiting, J. B. (2008). The role of appraisal distortion, contempt, and morality in couple conflict: A grounded theory. Journal of Marital and Family Therapy, 34(1), 44-57. doi:10.1111/j.17520606.2008.00052.x

Zordan, E. P., Wagner, A., \& Mosmann, C. (2012). O perfil de casais que vivenciam divórcios consensuais e litigiosos: Uma análise das demandas judiciais. Psico-USF, 17(2), 185-194. Recuperado em http:/www.scielo.br/scielo.php?script $=$ sci arttext\&pid $=$ S1413-82712012000200002
Recebido: 18/09/2014

$1^{a}$ revisão: 03/02/2015

$2^{a}$ revisão: 04/04/2015 Aceite final: 15/04/2015 